\title{
Initial experience of complete laparoscopic radical nephroureterectomy combined with transvesical laparoscopic excision of distal ureter in patients with upper urinary tract cancer
}

\author{
Makito Miyake* (D), Nobutaka Nishimura, Katsuya Aoki, Chihiro Ohmori, Takuto Shimizu, Takuya Owari, Shunta Hori, \\ Yosuke Morizawa, Daisuke Gotoh, Yasushi Nakai, Satoshi Anai, Kazumasa Torimoto, Nobumichi Tanaka and \\ Kiyohide Fujimoto
}

\begin{abstract}
Background: Selecting the treatment procedure for cancer patients is a challenging task. We report our initial experience of complete laparoscopic radical nephroureterectomy (RNU) for patients with upper urinary tract urothelial cancer (UTUC).
\end{abstract}

Methods: A total of four patients with UTUC underwent complete laparoscopic RNU combined with transvesical laparoscopic excision of the distal ureter using three 5-mm ports. Transvaginal specimen extraction was applied in female patients to reduce incisional pain and improve cosmesis. Peri-operative complications were evaluated using the Clavien-Dindo classification system. Postoperative pain was evaluated during hospitalization using a numeric pain rating scale (scales of 1 to 10). Patients who underwent retroperitoneal laparoscopic surgery combined with open excision of the distal ureter during the same period were included as a control group (conventional RNU, consisting of laparoscopic nephrectomy combined with open bladder cuff excision) for pain scale evaluation.

Results: The novel surgery was successfully completed for all four patients (two males and two females). The mean pneumoperitoneum time for retroperitoneoscopic nephroureterectomy and specimen extraction was 174 min, while the mean pneumovesicum time for the ureteral orifice excision was $88 \mathrm{~min}$. One male patient had bladder leakage at the suture site of the bladder wall, which lasted for 2 weeks. No patient experienced recurrent disease during the follow-up period (median, 10 months). Mild to moderate pain lasted for 5 or 6 days after RNU. A couple of days after surgery, the numeric pain rating scale of complete laparoscopic RNU and conventional RNU group reached its peak level at $3.0 \pm 1.8$ and $5.3 \pm 2.8$, respectively. There was no statistical difference in the degree of postoperative pain $(P=0.31)$.

(Continued on next page)

\footnotetext{
* Correspondence: makitomiyake@yahoo.co.jp

Department of Urology, Nara Medical University, 840 Shijo-cho, Kashihara,

Nara 634-8522, Japan
}

(c) The Author(s). 2020 Open Access This article is licensed under a Creative Commons Attribution 4.0 International License, which permits use, sharing, adaptation, distribution and reproduction in any medium or format, as long as you give appropriate credit to the original author(s) and the source, provide a link to the Creative Commons licence, and indicate if changes were made. The images or other third party material in this article are included in the article's Creative Commons licence, unless indicated otherwise in a credit line to the material. If material is not included in the article's Creative Commons licence and your intended use is not permitted by statutory regulation or exceeds the permitted use, you will need to obtain permission directly from the copyright holder. To view a copy of this licence, visit http://creativecommons.org/licenses/by/4.0/ The Creative Commons Public Domain Dedication waiver (http://creativecommons.org/publicdomain/zero/1.0/) applies to the data made available in this article, unless otherwise stated in a credit line to the data. 
(Continued from previous page)

Conclusions: We described our initial experience and outcome of complete laparoscopic RNU for UTUC. Further experience and research are required to determine whether this advanced laparoscopic technique yields better outcomes and has true clinical value.

Keywords: Upper urinary tract urothelial cancer, Transvesical laparoscopy, Complete laparoscopy, Pneumovesicum, Numeric pain rating scale

\section{Introduction}

Selecting the best treatment procedure for cancer patients in the clinical practice is a challenging task. Radical nephroureterectomy (RNU) with complete removal of the distal ureter, including ureteral orifice (bladder cuff excision), is the standard surgical treatment for upper urinary tract urothelial cancer (UTUC) [1, 2]. Although open RNU was commonly performed before 1990, minimally invasive laparoscopic surgery has rapidly evolved since 1991, with the first reports of laparoscopic RNU being reported by Clayman et al. [3]. Despite the lack of welldesigned prospective randomized control trials, metaanalysis-based comparisons between laparoscopic RNU and its open counterpart demonstrated that both offer equivalent outcomes in terms of oncological efficacy, perioperative safety, and mortality $[4,5]$.

To date, laparoscopic RNU has been broadly accepted by urologists mainly due to reduced postoperative pain and improved cosmetic results. Many urologists select a combination of excision of the kidney and upper ureter by retroperitoneoscopic approach and bladder cuff excision by lower abdominal incision-open surgery. This combination is not a complete laparoscopic surgery and thus is not a minimally invasive method. The best method for complete laparoscopic RNU for patients with UTUC has not yet been established. The approaches for bladder cuff excision are still controversial and include intravesical, extravesical, and transurethral incisions [6]. The extravesical technique carries a potential risk of residual tumor at the distal intramural part of the ureter, while the intravesical technique carries the risk of tumor cell dissemination to the outside of the bladder.

Here, we report the initial experience of complete laparoscopic nephroureterectomy with transvesical laparoscopic excision of the distal ureter using three ports in patients with UTUC. The transvesical laparoscopic approach is an alternative to open surgery in children with vesicoureteral reflux and bladder diverticulum [7, 8]. Transvaginal specimen extraction was applied in the case of female patients to reduce incisional pain and improve cosmesis.

\section{Patients and methods}

\section{Clinical data}

This research was approved by the ethics committee of the Nara Medical University, and all participants provided informed consent (reference ID: 1256 and 1719). The date of ethical approval was May 30, 2017, which is prior to the first surgery performed. We prospectively selected the patients and gave full information regarding the novel surgical method. All patients underwent dynamic computed tomography $(\mathrm{CT})$ or $\mathrm{CT}$ urography before surgery to determine tumor location and size. The main exclusion criteria were (1) tumor of the distal ureter, (2) advanced tumor detected by CT (suspected T3/T4 disease or node-positive disease), (3) contraindications to laparoscopic surgery, or (4) concurrent bladder tumor.

We conducted a review of four patients with UTUC who underwent complete laparoscopic nephroureterectomy with transvesical laparoscopic bladder cuff excision between January 2018 and December 2019. All surgeries were performed by a single laparoscopically experienced surgeon (M. Miyake). Peri-operative complications were objectively evaluated using the Clavien-Dindo classification system [9]. This system includes seven grades (I, II, IIIa, IIIb, IVa, IVb, and V). The degree of postoperative pain was evaluated every day during hospitalization and on the first outpatient visit day using a numeric pain rating scale (NPRS), where 0 indicates no pain and 10 indicates the worst imaginable pain [10]. Four patients who underwent conventional retroperitoneal laparoscopic surgery combined with an open bladder cuff using a lower abdominal midline incision, by the same surgeon (M. Miyake), during the same period, were included as a control group for pain scale evaluation.

\section{Surgical procedure for complete laparoscopic RNU}

An operation video demonstrating the surgical procedures is given in Supplementary Video S1. A diagram of each step of the surgical procedure is depicted in Fig. 1. Under general anesthesia, each patient was placed in a lateral decubitus position with the cancerous side up for a retroperitoneoscopic nephrectomy. The retroperitoneal cavity was dilated with a retroperitoneal balloon and maintained with $8 \mathrm{mmHg} \mathrm{CO}_{2}$ of insufflation pressure. We carried out conventional retroperitoneal approach with four ports as shown in Fig. 2a: a flexible endoscope (camera) trocar, 12-mm trocar, 5-mm trocar, and auxiliary 5-mm trocar. The procedure includes the standard nephrectomy using laparoscopic monopolar 


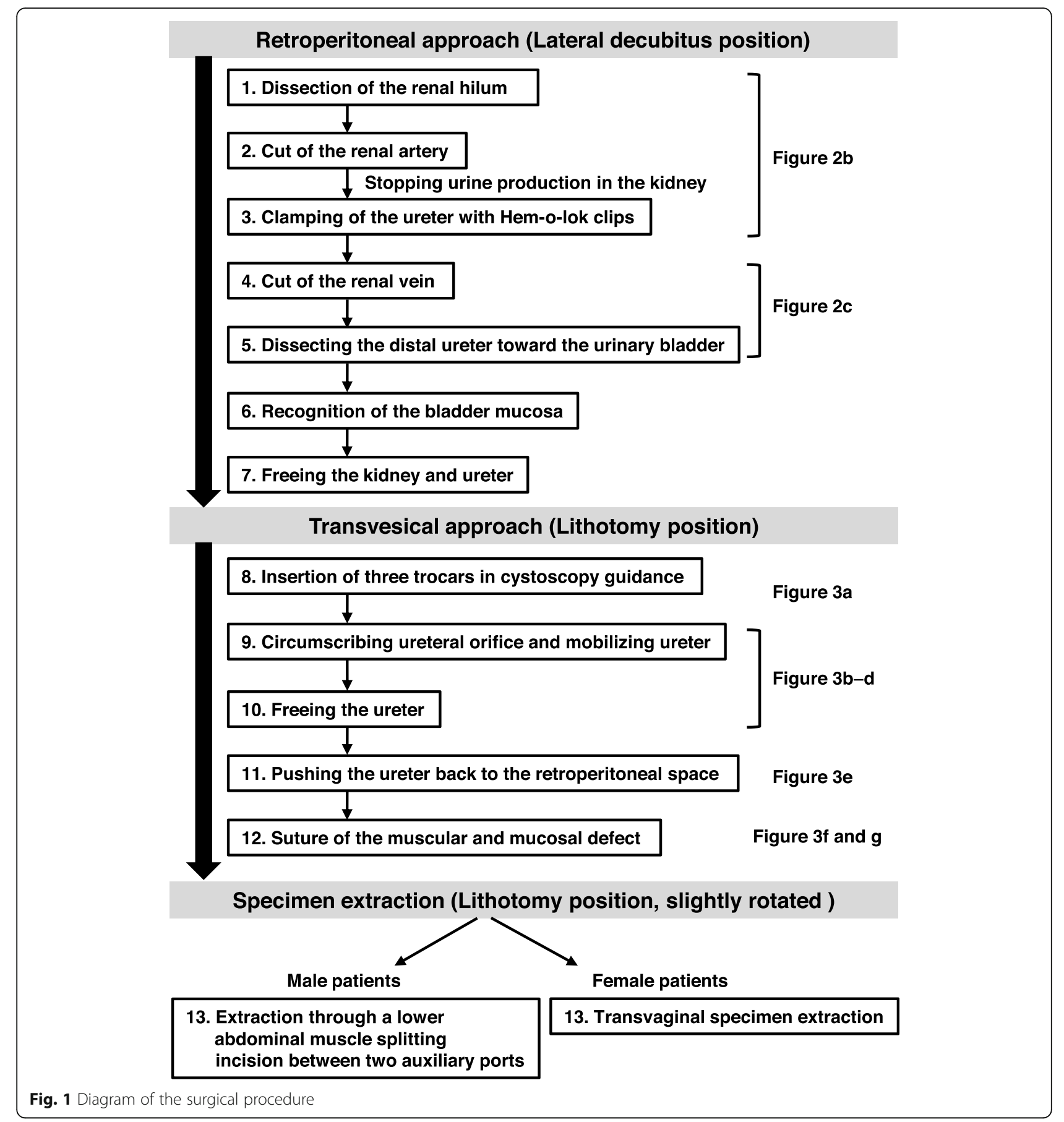

scissors (e.g., AESCULAP ${ }^{\oplus}$ laparoscopic instruments), LigaSure $^{\mathrm{TM}}$ Maryland jaw sealer (Covidien Japan, Tokyo, Japan), and Hem-o-lok ${ }^{\odot}$ clips [11]. The renal artery was secured with a size $\mathrm{L}$ clip, followed by clamping of the ureter at the distal area with a size L clip or ML clip after stopping urine production in the kidney (Fig. 2b). Then, the renal vein was secured with a size XL clip, and the kidney was completely freed. The adrenal gland was retained in all cases.
A 5-mm trocar was added to the ipsilateral pelvic area to facilitate a wide operation space in the phase of dissection of the distal ureter (Fig. 2a, red triangle). The ureter was dissected under the common iliac artery to the bladder. The distal ureter was dissected maximally, and the urinary bladder remained unclosed during this phase (Fig. 2c). During the retroperitoneoscopic procedure, the ureter was not cut to enable complete en bloc removal of the kidney and ureter. 
a

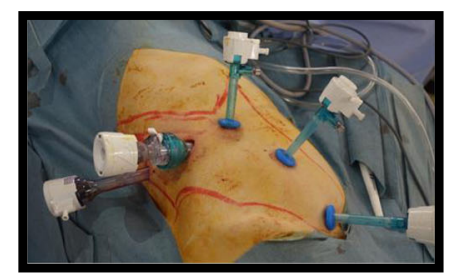

Lateral decubitus position

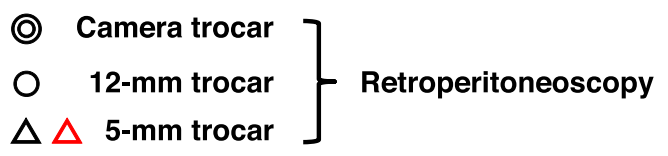

- 5-mm trocar — Transvesical laparoscopy

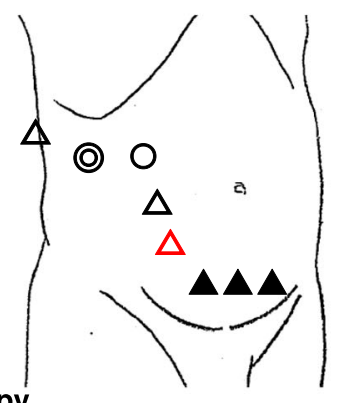

b

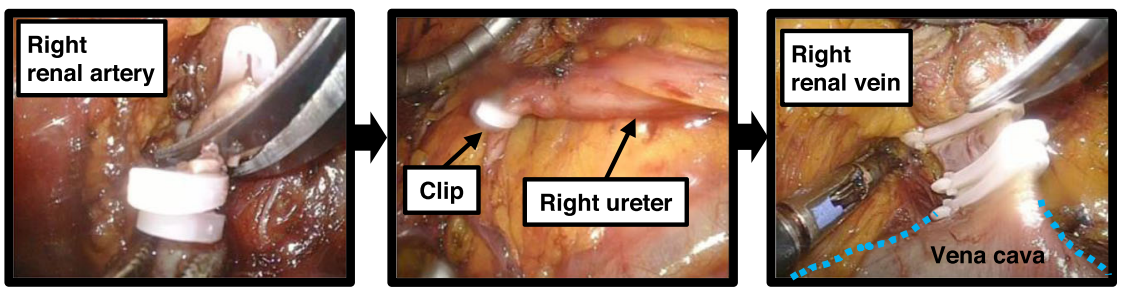

C
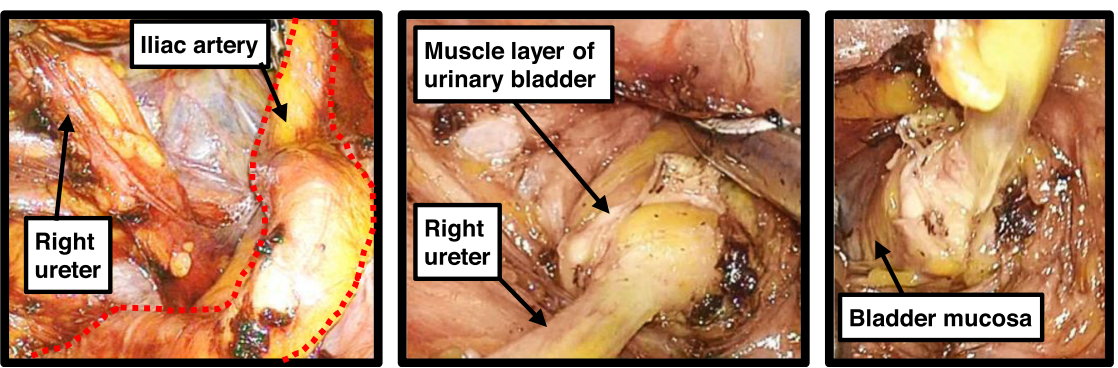

Fig. 2 Representative image (case 4 in Table 1) of a patient undergoing complete laparoscopic nephroureterectomy. a Trocar positions for nephroureterectomy of a right-side UTUC. The lower-level auxiliary port (red open triangle) is added to provide a wide surgical view of the pelvic area. Suprapubic ports (black triangles) are used for transvesical laparoscopic bladder cuff excision. b The cut of the renal artery, clamping of the ureter, and cut of the renal vein were performed with Hem-o-lok ${ }^{\circledR}$ clips.c Pulling up the proximal ureter to assist in dissecting the distal ureter toward the urinary bladder. When the junction of the ureter and bladder is exposed, the muscle layer is cut off, followed by recognition of the bladder mucosa

Next, the patient was changed to the lithotomy position for transvesical laparoscopic bladder cuff excision. The operation was restarted with cystoscopy and performed according to the procedure reported by Yeung et al. [12]. The surgeon stands on the patient's left side. Under cystoscopic guidance, three 5-mm trocars (Kii Advanced Fixation Sleeve; Applied Medical, Rancho Santa Margarita, CA, USA) were placed from the suprapubic region into the bladder (Fig. 3a). Under $8 \mathrm{mmHg}$ $\mathrm{CO}_{2}$ of pneumovesicum pressure, the ureterovesical junction and Waldeyer's sheath were excised with a 3$\mathrm{mm}$ laparoscopic monopolar scissors until the paravesical adipose tissue was visible (Fig. 3b-d). After completely mobilizing the ureter, the ureter was pushed back to the retroperitoneal space (Fig. 3e). Then, the muscular defect and mucosal defect in the ureteral hiatus were sutured intravesically (Fig. 3f, g). Intravesical trocars were removed under endoscopic vision without suturing the bladder wall. Each port site entry wound was closed with a 4-0 PDSII monocryl suture (Ethicon, NJ, USA).

In female patients, the specimen was extracted transvaginally in a bag (Fig. 4a). In male patients, the specimen was extracted in a bag through a lower abdominal muscle splitting incision between the two auxiliary ports. A pelvic drain tube was placed through the pelvic auxiliary port.

\section{Control group undergoing conventional RNU}

Conventional surgery consists of laparoscopic nephrectomy with open bladder cuff excision. In the retroperitoneoscopic phase, the ureter was freed to the bifurcation level of iliac vessels along the level of the lower pole of the kidney. Next, the patient was placed in the supine position for open bladder cuff excision. An 8-10-cm 
a

Lithotomy position

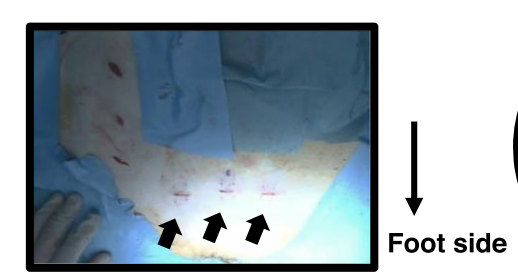

Trocar insertion in cystoscopy guidance
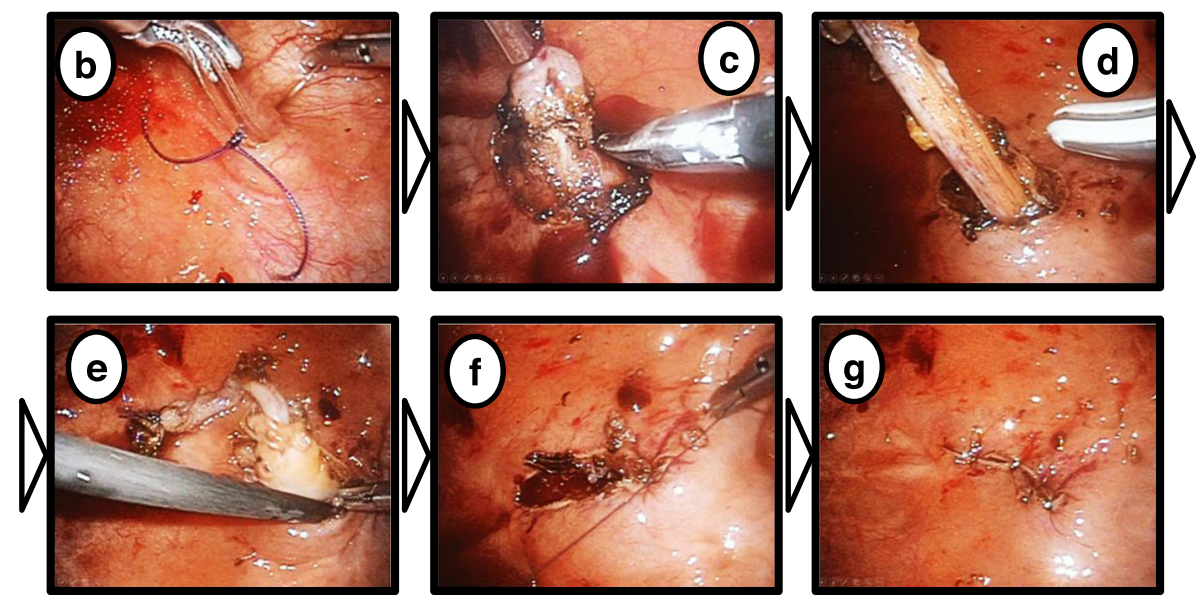

Fig. 3 Procedure of transvesical laparoscopic bladder cuff excision. a Postoperative wound for the suprapubic three ports is shown (black arrows). The bladder was distended with $400-500 \mathrm{~mL}$ of saline. A total of three 5-mm trocars were placed at the bladder dome and on both sides of the lateral wall of the distended bladder under cystoscopy guidance. A 3-0 monofilament traction suture is passed percutaneously through the bladder walls to prevent the bladder wall from falling away from the abdominal wall. b A 4-cm-long segment of an 8Fr pediatric feeding tube is inserted into the ipsilateral ureter to facilitate ureteral mobilization and dissection and secured by a 5-zero monofilament suture. c Circumscribing ureteral orifice and mobilizing ureter using fine 3-mm endoscopic scissors. $\mathbf{d}$ Traction on the ureteric catheter and cut of fibrovascular tissue surrounding the ureter to free it. e The ureter is pushed back to the retroperitoneal space. $\mathbf{f}$ The muscular defect and mucosal defect in the ureteral hiatus are sutured intravesically using 5-zero absorbable monofilament sutures, usually with an extracorporeal knot-tying technique. $\mathbf{g}$ Complete suturing of the bladder wall defect

midline incision was made on the lower abdomen. After extracting the kidney from the body, pulling the ureter exposed the bladder, thus facilitating excision of the bladder cuff. The remaining junction of the ureter and bladder was cut off, and the bladder wall was sutured with a 3-0 Vicryl suture. A pelvic drain tube was placed, and the incision was closed.

\section{Follow-up after the RNU}

A urinary catheter was left for approximately a week and removed after cystography. Cystoscopy and chest/abdomen/pelvis CT scans were performed approximately every 3 months for 2 years after the RNU, every 6 months from year 2 to 5 , and annually thereafter.

\section{Results}

Four patients undergoing complete laparoscopic RNU and four patients undergoing conventional RNU during the same period were included in this study. All four patients successfully underwent complete laparoscopic
RNU. Clinicopathological background and surgical information are shown in Tables 1 and 2, respectively. There were no statistically significant differences in age, length of hospital stay, and follow-up duration between the two groups.

Pneumoperitoneum time was longer in the complete laparoscopic RNU group compared to the conventional RNU group ( $174 \mathrm{~min}$ vs $99 \mathrm{~min} ; P=0.029$ ). The pneumovesicum time of the second patient was longer than that of the other three patients because he had a history of radiotherapy for localized prostate cancer. His bladder wall did not have elasticity, and so it took longer and it was more difficult to suture the bladder wall. Cystoradiography was performed on postoperative day 6 or 7 , and the urethral catheters were removed. No leakage at the transvesical port was observed. However, in the second patient, bladder leakage at the suture site of the bladder wall lasted for 2 weeks. Repeat cystoradiography demonstrated resolution of the leak, followed by removal of the urethral catheter. All patients were pathologically 


\section{a}

Case 4: 80 years-old female, right upper ureteral cancer
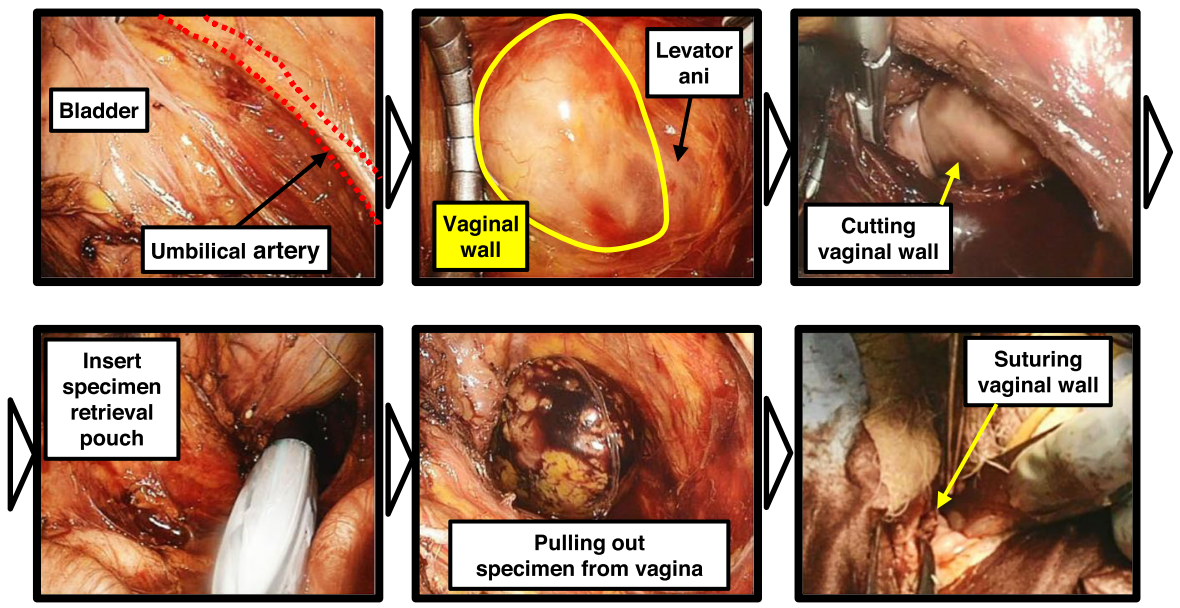

b

Case 2: 78 years-old male, left renal pelvic cancer
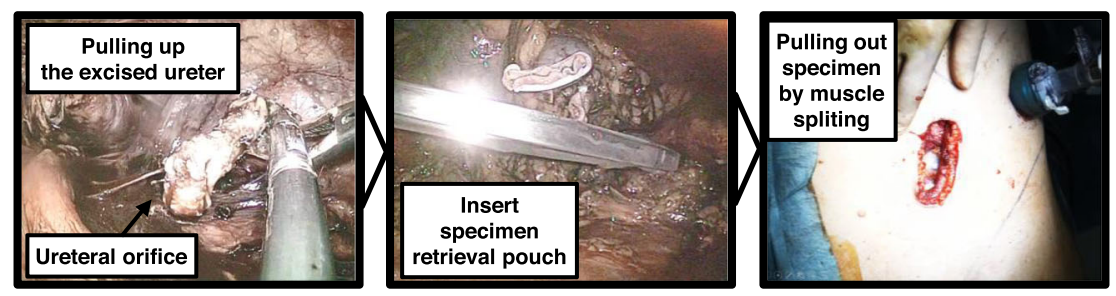

C
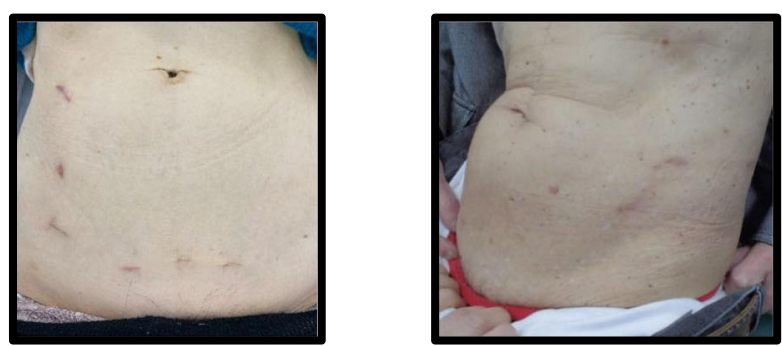

Case 4 : one month after RNU

\section{Case 2 : 15 months after RNU}

Fig. 4 Procedure of en bloc tissue extraction: two representative cases. a Transvaginal specimen extraction was applied in an 80-year-old patient with right upper ureter cancer. The specimen was packed in EndoCatch ${ }^{T M} \|$ specimen retrieval pouch (Medtoronic, Minneapolis, MN, USA) through the vaginal side wall. After pulling out the specimen, the vaginal wall was closed with absorbable surgical suture. b In male patients, the specimen was extracted in a retrieval pouch through a lower abdominal muscle splitting incision between two auxiliary ports. $\mathbf{c}$ Photographs of postoperative wounds in two representative cases

diagnosed with urothelial carcinoma, and none of the patients experienced recurrent disease during the followup (range, 3-15 months).

Time-course changes in the NPRS of the complete laparoscopic RNU group $(n=4)$ and conventional RNU group $(n=4)$ are plotted in Fig. 5. Generally, mild to moderate pain lasted for 5 or 6 days after RNU. A couple of days after surgery, the NPRS of the complete laparoscopic RNU and conventional RNU groups reached peak levels at $3.0 \pm 1.8$ and $5.3 \pm 2.8$, respectively. There was no statistical difference in the degree of postoperative pain $(P=0.31$, Mann-Whitney $U$ test $)$ in our study cohort.

\section{Discussion}

Recent advances in laparoscopic skills and devices have enabled a safe and minimally invasive procedure by visual magnification, providing accurate suture and anastomosis. In this study, we report the initial experience of complete laparoscopic RNU with transvesical laparoscopic bladder cuff excision in patients with UTUC. 


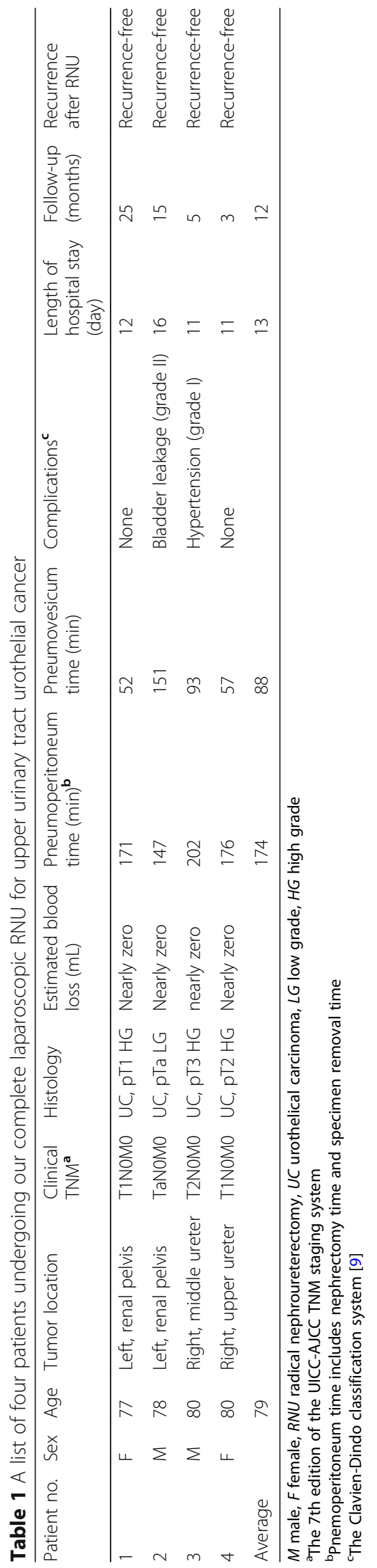




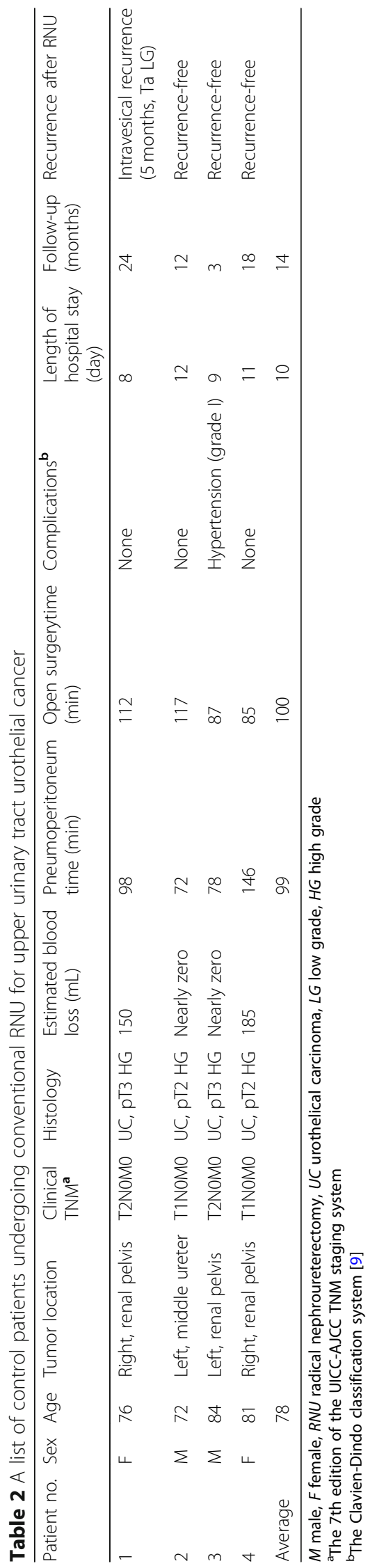


Numeric Pain Rating Scale (NPRS) : 0 - 10

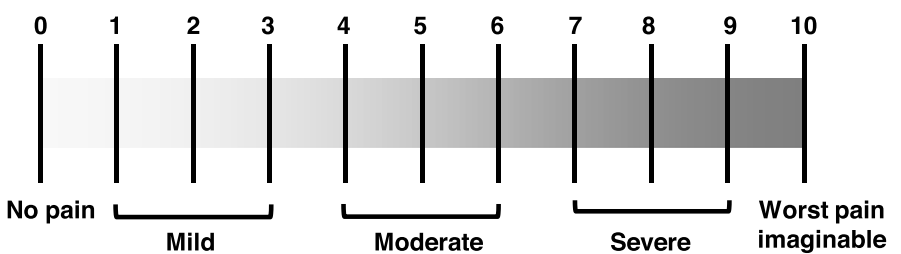

Complete laparoscopic RNU

Conventional RNU
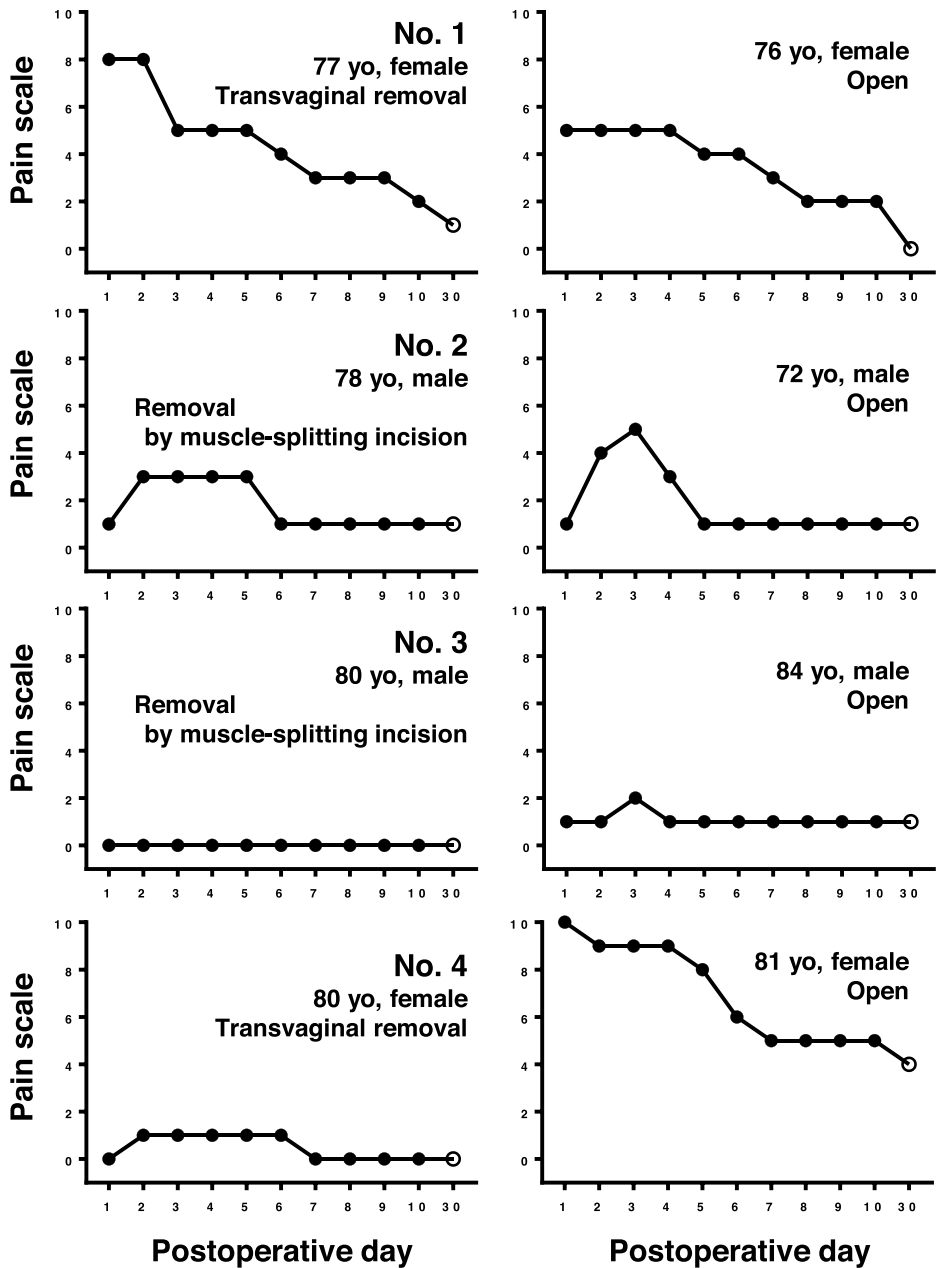

Fig. 5 Time-course change of pain scale of complete laparoscopic nephroureterectomy and conventional nephroureterectomy. Postoperative pain was assessed using a numerical pain rating scale (NPRS) every day during the hospitalization and at the first outpatient visit day (indicated with "day 30"). The data included four patients undergoing complete laparoscopic radical nephroureterectomy (RNU), as shown in Table 1, and four patients undergoing the conventional retroperitoneal laparoscopic nephrectomy combined with an open bladder cuff by the same surgeon, during the same period, as shown in Table 2

Several techniques, especially in excision of the distal ureter, have been reported to date [13-16]. However, we should carefully consider the application of laparoscopic surgery in patients with UTUC, which is known as one of the most aggressive malignancies. During the followup, no patients experienced any recurrent disease including intravesical, extravesical, distant, and vaginal recurrence. As to the learning curve, apparent progress was not observed in terms of operation time. Longer follow-up and larger sample size are mandatory to clarify the real benefit of this surgical method.

Maximal effort should be made to ameliorate postoperative pain, shorten hospitalization stay, prevent perioperative complications, and maintain cosmesis. Based 
on the initial experience of our four patients, we considered our novel trial of complete laparoscopic RNU as an acceptable method (Table 1). The intravesical space is limited and can be continually obscured by effluxing urine. To counter this problem, the retroperitoneal procedure, including ligation of the renal artery and ureter, proceeds the transvesical laparoscopic procedure in our operation strategy. This can provide an additional possible advantage that occluding the ureter results in decreased risk of dissemination of tumor cells from the upper urinary tract to the intravesical cavity and paravesical space. The biggest concern with transurethral excision of the distal ureter, the so-called pluck technique, is the risk of spillage of urine containing tumor cells [17].

In a case report by Sotelo et al. [16], they performed a transvesical single-site surgery enabling full-thickness incision of the bladder around the ureteral orifice, followed by a water-tight suture of the bladder wall defect. Transvesical single-site surgery requires insertion of a special device into the bladder and subsequent suture of the bladder anterior wall. In contrast to single-site surgery, the out method does not require suture of the bladder port site because intravesical trocars are thin (only $5 \mathrm{~mm}$ thickness). The technique for dissection of the intramural ureter and intracorporeal suturing seems to be challenging and time-consuming, even for a laparoscopically experienced surgeon. In our second patient (Table 1), the intravesical laparoscopic procedure took no less than $2.5 \mathrm{~h}$, mainly due to a history of radiotherapy for prostate cancer, which could induce ischemic conditions around the bladder trigon and decreased elasticity of the bladder wall. Eventually, he suffered from prolonged urethral catheterization. Based on this experience, a history of pelvic radiotherapy may be a potential contraindication for transvesical laparoscopic bladder cuff excision.

We applied three 5-mm trocars to the intravesical space, which may lead to a risk of port-site recurrence. No patients experienced port-site recurrence during the follow-up period in our cohort. Other options for laparoscopic excision of the distal ureter and bladder cuff are as follows: thermo-sealing system [18], cold excision of the bladder cuff with intracorporeal suturing $[14,15]$, modification with a bulldog clamp [19], purse-string technique [20], and robot-assisted surgery [21]. As pointed out previously, some extravesical approaches have the potential risk of failing to resect the intramural ureter. Our peumovesicoscopic bladder cuff excision ensures complete resection of the ureteral orifice, intramural ureter, and ureterovesical junction. This advantage strengthens our novel method. The transvesical laparoscopic bladder cuff technique has not yet been standardized.

We applied transvaginal specimen extraction in two female patients (cases. 1 and 4 in Table 1). We believe that the potential benefits of our method over conventional RNU could include decreased incisional pain, reduction in postoperative pain, and improved cosmesis. Since the first preclinical model of natural orifice transluminal endoscopic surgery (NOTES) nephrectomy in the urologic field [22], this approach has attracted much interest from urologists. Transvaginal NOTES has been introduced into donor nephrectomy and nephrectomy for renal infection, renal calculus, and renal malignancies [23-26]. In the literature, the multiaccess alternative of pure NOTES is often mentioned as "Hybrid NOTES," in which standard laparoscopic nephrectomy is performed and the vagina is only utilized for specimen extraction.

This study has several limitations. Given that the decision between complete laparoscopic RNU or conventional RNU was made by physicians rather than through randomization, there is a potential selection bias. Second, the sample size was small, with only four patients in the complete laparoscopic RNU group and four patients in the conventional RNU group, and the follow-up time was short. We could not make conclusion regarding oncological outcome and benefit. However, we believe the potential of functional outcomes including decreased postoperative pain and improved cosmesis. This report can emphasize on the safety and feasibility of the new surgical method, which lead to our prospective multi-institutional trial in the near future. Third, no assessment was conducted regarding patient-reported outcomes such as health-related quality of life and sexual satisfaction questionnaire pre- and post-operatively, especially in female patients.

\section{Conclusion}

We reviewed our experience with complete laparoscopic RNU for patients with UTUC. Although there have been many reports on the feasibility of complete laparoscopic surgery, few studies have reported on transvesical threeport bladder cuff excision. Further experience and research are mandatory to determine whether this advanced laparoscopic technique yields better outcomes and has true clinical value.

\section{Supplementary information}

Supplementary information accompanies this paper at https://doi.org/10. 1186/s12957-020-01872-1.

Additional file 1: Supplementary Video S1: Operation video demonstrating the surgical procedures.

Abbreviations

CT: Computed tomography; NOTES: Natural orifice translumenal endoscopic surgery; NPRS: Numeric pain rating scale; RNU: Radical nephroureterectomy; UTUC: Upper urinary tract urothelial cancer 


\section{Acknowledgements}

We would like to thank Editage (www.editage.jp) for English language editing.

\section{Authors' contributions}

Conceptualization, M.M. and K.A. Methodology, N.N., C.O., T.S., and T.O. Investigation, S.H., Y.M., and D.G. Data curation, M.M. and S.A. Writing_original draft preparation, M.M. Writing-review and editing, K.A., K.T., and K.F. Visualization, M.M. Supervision, N.T. The author(s) read and approved the final manuscript.

\section{Funding}

No specific funding in this study

\section{Availability of data and materials}

All data generated or analyzed during this study are included in this published article and reference articles.

\section{Ethics approval and consent to participate}

This study was approved by the ethics committee of the Nara Medical University (reference ID: 1256 and 1966). Informed consent to participate in the study was obtained from all participants. The study was conducted in compliance with the study's protocol and in accordance with the provisions of the Declaration of Helsinki (2013).

\section{Consent for publication}

Consent for publication of individual patients was obtained from the participants.

\section{Competing interests}

The authors declare that they have no competing interests.

Received: 16 March 2020 Accepted: 6 May 2020

Published online: 25 May 2020

\section{References}

1. Miyake $M$, Tatsumi $Y$, Fujimoto $K$, et al. Changes in oncological outcomes after radical nephroureterectomy in patients with upper urinary tract urothelial carcinoma treated in the last two decades: a retrospective analysis based on a multicenter collaborative study. Jpn J Clin Oncol. 2016;46:1148-55.

2. Roupret M, Babjuk M, Comperat E, et al. European Association of Urology Guidelines on upper urinary tract urothelial cell carcinoma: 2015 update. Eur Urol. 2015;68:868-79.

3. Clayman RV, Kavoussi LR, Figenshau RS, et al. Laparoscopic nephroureterectomy: initial clinical case report. J Laparoendosc Surg. 1991;1: 343-9.

4. $\quad \mathrm{Ni}$ S, Tao W, Chen Q, et al. Laparoscopic versus open nephroureterectomy for the treatment of upper urinary tract urothelial carcinoma: a systematic review and cumulative analysis of comparative studies. Eur Urol. 2012;61: $1142-53$.

5. Zhang $\mathrm{X}$, Wang $\mathrm{K}, \mathrm{Ma}$ J, et al. Total laparoscopic nephroureterectomy for upper urinary tract urothelial carcinoma under a single surgical position. World J Surg Oncol. 2019;17:65.

6. Li WM, Shen JT, Li CC, et al. Oncologic outcomes following three different approaches to the distal ureter and bladder cuff in nephroureterectomy for primary upper urinary tract urothelial carcinoma. Eur Urol. 2010;57:963-9.

7. Casale P, Kojima Y. Robotic-assisted laparoscopic surgery in pediatric urology: an update. Scand J Surg. 2009;98:110-9.

8. Badawy H, Eid A, Hassouna M, et al. Pneumovesicoscopic diverticulectomy in children and adolescents: is open surgery still indicated? J Pediatr Urol. 2008:4:146-9.

9. Dindo D, Demartines N, Clavien PA. Classification of surgical complications: a new proposal with evaluation in a cohort of 6336 patients and results of a survey. Ann Surg. 2004;240:205-13.

10. Shigematsu-Locatelli M, Kawano T, Kitamura S, et al. Does preoperative patient's estimated acceptable pain affect the satisfaction with postoperative pain management? JA Clin Rep. 2017;3:5.

11. Casale P, Pomara G, Simone M, et al. Hem-o-lok clips to control both the artery and the vein during laparoscopic nephrectomy: personal experience and review of the literature. J Endourol. 2007;21:915-8.
12. Yeung CK, Sihoe JD, Borzi PA. Endoscopic cross-trigonal ureteral reimplantation under carbon dioxide bladder insufflation: a novel technique. J Endourol. 2005;19:295-9.

13. Hora M, Eret V, Urge T, et al. Complete laparoscopic nephroureterectomy with intravesical lockable clip. Cent European J Urol. 2012;65:75-9.

14. Hemal AK, Kumar A, Gupta NP, et al. Retroperitoneal nephroureterectomy with excision of cuff of the bladder for upper urinary tract transitional cell carcinoma: comparison of laparoscopic and open surgery with long-term follow-up. World J Urol. 2008;26:381-6.

15. Hattori R, Yoshino Y, Komatsu T, et al. Pure laparoscopic complete excision of distal ureter with a bladder cuff for upper tract urothelial carcinoma. World J Urol. 2009;27:253-8.

16. Sotelo R, Ramírez D, Carmona O, et al. A novel technique for distal ureterectomy and bladder cuff excision. Actas Urol Esp. 2011;35:168-74.

17. Srirangam SJ, van Cleynenbreugel B, van Poppel H. Laparoscopic nephroureterectomy: the distal ureteral dilemma. Adv Urol. 2009, 2019; 316807

18. Hora M, Stránský P, Eret V, et al. Complete laparoscopic nephroureterectomy with thermo-sealing system. CEJU. 2010;63:77-81.

19. Cho HJ, Kim SJ, Yoon Bl, et al. A novel bulldog clamp technique for management of a distal ureter and bladder cuff during laparoscopic nephroureterectomy. J Endourol. 2010;24:1719-20.

20. Shoma AM. Purse-string technique for laparoscopic excision of a bladder mucosal cuff in patients with transitional cell carcinoma of the upper urinary tract: initial report with intermediate follow-up. BJU Int. 2009;104: $1505-9$.

21. Park SY, Neony W, Ham WS, et al. Initial experience of robotic nephroureterectomy: a hybrid-port technique. BJU Int. 2009:104:1718-21.

22. Gettman MT, Lotan Y, Napper CA, et al. Transvaginal laparoscopic nephrectomy: development and feasibility in the porcine model. Urology. 2002:59:446-50

23. Georgiopoulos I, Kallidonis P, Kyriazis I, et al. Hybrid transvaginal nephrectomy: development of our technique. Urology. 2014;84:99-104.

24. Guner Can M, Ozcan P, Hatipoglu S, et al. Laparoscopic-assisted live donor nephrectomy: a comparison of conventional and transvaginal routes for kidney extraction. Ann Transplant. 2015;20:634-8.

25. Xue Y, Zou X, Zhang G, et al. Transvaginal natural orifice transluminal endoscopic nephrectomy in a series of 63 cases: stepwise transition from hybrid to pure NOTES. Eur Urol. 2015;68:302-10.

26. Sener TE, Sahin B, Fichera $M$, et al. Does vaginal wall surgical trauma during hybrid transvaginal NOTES nephrectomy have traumatic effects on sexual functions? A prospective study. J Invest Surg. 2020;6:1-8. https://doi.org/10. 1080/08941939.2019.1710627 [Epub ahead of print].

\section{Publisher's Note}

Springer Nature remains neutral with regard to jurisdictional claims in published maps and institutional affiliations.

Ready to submit your research? Choose BMC and benefit from:

- fast, convenient online submission

- thorough peer review by experienced researchers in your field

- rapid publication on acceptance

- support for research data, including large and complex data types

- gold Open Access which fosters wider collaboration and increased citations

- maximum visibility for your research: over $100 \mathrm{M}$ website views per year

At $\mathrm{BMC}$, research is always in progress.

Learn more biomedcentral.com/submission 